\title{
Ecogeografía del género Sticta (Ascomycota liquenizados: Lobariaceae) en Colombia
}

\author{
Bibiana Moncada ${ }^{1}$, Jaime Aguirre ${ }^{2} \&$ Robert Lücking ${ }^{3}$ \\ 1. Licenciatura en Biología, Universidad Distrital Francisco José de Caldas, Cra. 4 No. 26D-54, Torre de Laboratorios, \\ Herbario, Bogotá, Colombia; lbmoncada@udistrital.edu.co, bibianamoncada@gmail.com \\ 2. Universidad Nacional de Colombia, Instituto de Ciencias Naturales, Carrera 45 No 26-85; jaguirrec@unal.edu.co \\ 3. Department of Botany, The Field Museum, 1400 South Lake Shore Drive, Chicago, Illinois 60605-2496, U.S.A.; \\ rlucking@fieldmuseum.org
}

Recibido 19-IX-2012. Corregido 10-IX-2013. Aceptado 16-X-2013.

\begin{abstract}
Ecogeography of the genus Sticta (lichenized Ascomycota: Lobariaceae) in Colombia. Colombia is a megadiverse country, but with a substantial gap in the taxonomic and ecological knowledge of fungi and lichens. Thus, the objective of the present study was an ecogeographical analysis of the Sticta species in Colombia. The data included macrodistribution (with respect to mountain ranges and life zones) and microhabitat preferences (light, substrate) and were obtained from field work and herbarium collection labels (completed by comparison with modern geographic and vegetation maps), for 103 species of Sticta currently recognized in Colombia (plus seven additional biotypes with different photobionts or reproductive mode). Using non-metric multidimensional scaling (NMS), correlations between ecogeographical variables and species were established, and ecotypes were delimited. Colombian species of Sticta showed distinct distribution patterns relative to altitude, mountain ranges, life zones, as well as light exposure and substrate, forming several distinct groups. Cyanobacterial species tend to have wider distribution ranges than green algal species. We concluded that the orogeny of the Northern Andes substantially affected speciation of the genus Sticta and its ecogeographical differentiation. Rev. Biol. Trop. 62 (1): 257-272. Epub 2014 March 01.
\end{abstract}

Key words: Sticta, life zones, patterns of distribution, subandine, andine, páramo, superpáramo.

Colombia es uno de los países megadiversos (Samper \& García, 2001; BenavidesMolineros, Castiblanco-A. \& Vivas-Segura, 2006; Bernal, Celis \& Gradstein, 2007). La alta biodiversidad se ve favorecida por la presencia de zonas biogeografícas con condiciones contrastantes, generando un sinúmero de ambientes que resultan en una gran variedad de flora, fauna y ecosistemas. Según Álvarez (2001), Colombia ocupa el primer lugar en aves (Renjifo, Franco-Maya, Amaya-Espinel, Kattan \& López-Lanús, 2002; Andrade-C., 2011), segundo en plantas vasculares, lepidópteros y anfibios (Bernal, Celis \& Gradstein, 2007; Andrade-C., 2011) y quinto en mamíferos (Chaves \& Santamaría, 2006; RodríguezM., Alberico, Trujillo \& Jorgenson, 2006a).
Sin embargo, los grupos más diversos, como los artrópodos y los hongos (Hawksworth, 1991, 2001), han sido muy poco estudiados, y su diversidad en Colombia es virtualmente desconocida (Amat, Andrade-C. \& Amat, 2007). Esto incluye a los líquenes, hongos que establecen una simbiosis con algas verdes o cianobacterias (Nash, 2008). Lücking, Rivas Plata, Chaves, Umaña y Sipman (2009) estiman para Colombia un total de 3600 especies, de las cuales solo 1562 han sido informadas para el país (Aguirre-C. \& Rangel-Ch., 2008a; Sipman, Hekking \& Aguirre-C., 2008), correspondiendo a un $43 \%$ de la diversidad estimada.

Un $47 \%$ del área total de Colombia está cubierta por bosques naturales, $19 \%$ por otros tipos de vegetación como sabanas, zonas 
áridas, humedales aguas continentales y picos de nieve y $34 \%$ se encuentran bajo uso agrícola, asentamientos urbanos y procesos de colonización (Samper \& García, 2001), poneniendo en peligro la conservación de la extraordinaria biodiversidad del país. Una de las causas más importantes que hacen de Colombia un país megadiverso son los procesos de la orogenia andina, que permitieron el movimiento en altura de las montañas y la formación de los tres ramales de la Cordillera de los Andes, afectando la distribución espacial y especiación de los organismos. Seguido a estos procesos ocurrieron los cambios climáticos periódicos que dieron como resultado las modificaciones de la cobertura vegetal desde hace 25 millones de años hasta el presente (Hernández, Walschburger, Ortiz \& Hurtado, 1992).

Si el conocimiento de la diversidad de los líquenes tropicales es escaso, menos se sabe de su ecología y biogeografía. Estudios filogenéticos recientes sugieren que el paradigma que muchas especies tienen distribución pantropical (Galloway, 1979; Lücking, 2003; HerreraCampos et al., 2004; Feuerer \& Hawksworth, 2007; Aptroot \& Iqbal, 2011) es incorrecto y que la mayoría tienen rangos de distribución bien definidos (Molina, Crespo, Blanco, Lumbsch \& Hawksworth, 2004; Tehler, Irestedt, Wedin \& Ertz, 2010; Del Prado et al., 2006; Moncada, 2012; Moncada, Lücking \& Suárez, 2013), aumentando la posibilidad de que especies sean endémicas e incrementando el valor potencial de sus recursos genéticos y bioquímicos. Existen pocos estudios cuantitativos sobre la ecología de líquenes tropicales, y a menudo aplican una taxonomía superficial que no refleja conceptos apropiados que permitan delimitar especies o excluyen grupos taxonómicamente difíciles (Kelly et al., 2004; Holz \& Gradstein, 2005; Boonpragop \& Polyiam, 2007; Dyer \& Letourneau, 2007). Entre las excepciones están los estudios de los líquenes foliícolas de Costa Rica por Lücking (1998a, 1999a-c), los líquenes corticícolas de los llanos de Venezuela Komposch y Hafellner (2000, 2002, 2003), los líquenes del sur de Brasil (Martins, 2006) y los microlíquenes corticícolas del nordeste de Brasil (Cáceres, Lücking \& Rambold, 2007, 2008).

Las investigaciones relacionadas con la ecología y biogeografía de líquenes tropicales en Colombia incluyen a Wolf (1993a, b), Aguirre-C. y Sipman (2004), Aguirre-C. y RangelCh. (2008a, b), Sipman (1984, 1989, 1992, 1995, 2006), Sipman y Cleef (1979), Soto y Bolaños (2010), Simijaca (2011) y Soto-M., Lücking y Bolaños (2012). Wolf, cuyo trabajo estuvo enfocado en los cambios de biodiversidad y composición de líquenes y otros epífitos a lo largo de un transecto altitudinal en la Cordillera Central, es considerado uno de los pioneros en el uso de la técnica de escalar árboles y tomar muestras del dosel, donde se encuentra la mayor biodiversidad de los líquenes en los bosques tropicales húmedos (Wolf, 1993a, b; Komposch \& Hafellner, 2000, 2002, 2003).

En términos de ecogeografía, es decir la macrodistribución de líquenes con respecto a las regiones biogeográficas y zonas de vida, es escasa la información para Colombia (Aguirre \& Rangel-Ch., 2008a, b; Sipman et al., 2008). Recientemente, Rincón-Espitia, Aguirre-C. y Lücking (2011) presentaron un análisis de microlíquenes de la región del Caribe Colombiano, siendo el único estudio detallado para este grupo de líquenes hasta la fecha. Esto es desafortunado, ya que Colombia presenta condiciones ideales para hacer estudios biogeográficos y ecogeográficos de grupos de organismos, como ha ocurrido, por ejemplo, en plantas vasculares o insectos (Barney-D., 2011; Díaz, Solis \& Brochero, 2011).

El género Sticta es un grupo ideal para un estudio de ecogeografía en Colombia, ya que es principalmente andino y por lo tanto la ecogeografía de las especies debería verse afectada por los procesos de la orogenia andina. Especies de Sticta predominan en las zonas paramunas y bosques subandinos y andinos, donde cubren ramas y troncos de árboles y arbustos en grandes cantidades. Por asociarse en su mayoría con fotobiontes cianobacteriales, estos líquenes contribuyen a la fijación de nitrógeno atmosférico y sirven como fertilizantes naturales (Kelly \& Becker, 1975; Becker, 
1980; Green, Horstmann, Bonnett, Wilkins \& Silvester, 1980; Green \& Lange, 1991; Antoine, 2004; Benner, Conroy, Lunch, Toyoda \& Vitousek, 2007). Además, por su capacidad de retener grandes cantidades de agua, permiten balancear los efectos de la precipitación y generan un microclima húmedo sobre la vegetación terrestre aún tiempo después que la precipitación haya terminado (Green, Snelgar \& Wilkins, 1985; Guzmán, Quilhot \& Galloway, 1990; Green \& Lange, 1991; Beckett, 1995; Zotz, Büdel, Meyer, Zellner \& Lange, 1998). Por lo anterior, contribuyen para que los páramos sean fuentes indispensables de agua para áreas metropolitanas como lo es la ciudad de Bogotá (Luteyn, 1999; Crespo et al., 2010; De Brievre \& Calle, 2011).

El presente trabajo es un análisis de la ecogeografía del género Sticta en Colombia, aprovechando los datos obtenidos en su revisión sistemática y filogenética para el país y otros lugares (Moncada, 2012; Moncada \& Lücking, 2012; Moncada et al., 2013). El estudio se enfocó en la distribución de las especies con relación a las zonas de vida, comparando diferentes sistemas de clasificación de las mismas (Rangel-Ch., 1995, 2000; Poveda, Rojas, Rudas \& Rangel-Ch., 2004; Rodríguez, Armenteras, Morales \& Romero, 2006b) y considerando además, aspectos de microhábitat como luz relativa y sustrato.

\section{MATERIALES Y MÉTODOS}

Toma de datos: Se estudiaron 1765 ejemplares de Sticta de colecciones en los herbarios Colombianos ANDES, COAH, COL, CUVC, FAUC, HUA, UDBC y UPTC, y 848 ejemplares de recolecciones nuevas entre 2000 y 2012. Además, se incluyeron colecciones extraterritoriales, históricas y tipos disponibles en los herbarios: B, BM, F, FH, G, H, L, MEL, OXF, PC, RBGE, TUR, U, US y W.

Para cada muestra, se tomaron datos de campo o de las etiquetas (en caso de muestras de herbario) para establecer el rango de variables ambientales como: cordillera, altitud y zona de vida, sustrato y exposición a la luz en tres categorías: luz directa o zona abierta $=$ expuestas; mediana exposición, bosques o áreas cubiertas por ramas secundarias = intermedia; áreas con poca luminosidad o cubiertas con espesa vegetación $=$ sombreada. En el caso de información incompleta en ejemplares históricos, se trató de reconstruir los viajes históricos de los recolectores como, por ejemplo, Alexander Lindig (Nylander, 1863a, b, 1864, 1867; Stearn, 1968), para establecer una ubicación aproximada de cada lugar de la recolección.

Caracterización ecogeográfica: Para la caracterización de las áreas ecogeográficas se tuvo en cuenta el sistema de Rangel-Ch. (1995), que divide el territorio Colombiano en cinco grandes regiones (Andina o Cordillerana, Orinoquia, Amazonia, Chocó Biogeográfico y Caribe). Para la Región Andina (Cordillerana) la división, según altitud, en zonas de vida Tropical, Subandina, Andina y Páramo, además de diferenciar la Sierra Nevada de Santa Marta como Zona Septentrional. Adicionalmente, se hizo una caracterización basada en los pisos bioclimáticos propuesta por Rodríguez et al. (2006b), en la cual toma en cuenta la diferenciación según la orientación occidental u oriental de la cordillera correspondiente. Finalmente, se aplicó una caracterización basada en una combinación de los sistemas de clasificación mencionados (Rangel-Ch., 1995, 2000; Rodríguez et al., 2006b), diferenciando a su vez entre los pisos altitudinales como Altoandino, Subpáramo y Superpáramo (Cuadro 1).

Para las especies encontradas en los departamentos de Antioquia, Chocó, Valle del Cauca, Cauca y Nariño, se revisó su ubicación con el fin de determinar si pertenecían a la Región del Chocó Biogeográfico propuesta por Poveda et al. (2004).

Caracterización ecológica: Se usaron las variables de luz relativa, aplicando una clasificación simple en tres categorías como anteriormente definida (sombreada, intermedia, expuesta) y de sustrato, diferenciando seis tipos de sustrato: corteza, madera (generalmente 
CUADRO 1

Distribución altitudinal aproximada de pisos bioclimáticos para los Andes Colombianos, con referencia a Rangel-Ch. (1995) (A), Rodríguez et al. (2006b) (B), Rangel-Ch. (2000) (C) y una combinación de los diferentes sistemas de clasificación (D)

\begin{tabular}{|c|c|c|c|c|c|c|c|}
\hline \multirow{2}{*}{ Piso } & \multicolumn{2}{|c|}{ Cordillera Occidental } & \multicolumn{2}{|c|}{ Cordillera Central } & \multicolumn{2}{|c|}{ Cordillera Oriental } & \multirow{2}{*}{ Ref } \\
\hline & Vert. Occidental & Vert. Oriental & Vert. Occidental & Vert. Oriental & Vert. Occidental & Vert. Oriental & \\
\hline Tropical & $<1100 \mathrm{~m}$ & $<1100 \mathrm{~m}$ & $<1100 \mathrm{~m}$ & $<1100 \mathrm{~m}$ & $<1100 \mathrm{~m}$ & $<1100 \mathrm{~m}$ & A \\
\hline Subandino & $1100-2350 \mathrm{~m}$ & $1100-2350 \mathrm{~m}$ & $1100-2350 \mathrm{~m}$ & $1100-2350 \mathrm{~m}$ & $1100-2350 \mathrm{~m}$ & $1100-2350 \mathrm{~m}$ & A \\
\hline Andino & $2350-3500 \mathrm{~m}$ & $2350-3500 \mathrm{~m}$ & $2350-3500 \mathrm{~m}$ & $2350-3500 \mathrm{~m}$ & $2350-3500 \mathrm{~m}$ & $2350-3500 \mathrm{~m}$ & A \\
\hline Páramo & $>3500 \mathrm{~m}$ & $>3500 \mathrm{~m}$ & $>3500 \mathrm{~m}$ & $>3500 \mathrm{~m}$ & $>3500 \mathrm{~m}$ & $>3500 \mathrm{~m}$ & A \\
\hline Basal & $<1100 \mathrm{~m}$ & $<1200 \mathrm{~m}$ & $<1200 \mathrm{~m}$ & $<1200 \mathrm{~m}$ & $<1100 \mathrm{~m}$ & $<1100 \mathrm{~m}$ & B \\
\hline Subandino & $1100-2200 \mathrm{~m}$ & $1200-2400 \mathrm{~m}$ & $1200-2200 \mathrm{~m}$ & $1200-2200 \mathrm{~m}$ & $1050-2040 \mathrm{~m}$ & $1100-2300 \mathrm{~m}$ & B \\
\hline Andino & $2200-3300 \mathrm{~m}$ & $2200-3200 \mathrm{~m}$ & $2200-3500 \mathrm{~m}$ & $2200-3500 \mathrm{~m}$ & $2100-3200 \mathrm{~m}$ & $2100-3400 \mathrm{~m}$ & B \\
\hline Páramo & $>3300 \mathrm{~m}$ & $>3200 \mathrm{~m}$ & $>3500 \mathrm{~m}$ & $>3500 \mathrm{~m}$ & $>3200 \mathrm{~m}$ & $>3400 \mathrm{~m}$ & B \\
\hline \multicolumn{3}{|c|}{ Piso } & \multicolumn{4}{|c|}{ Todas las Cordilleras } & Ref \\
\hline \multicolumn{3}{|c|}{ Altoandina-Subpáramo o zona de ecotonía } & \multicolumn{2}{|c|}{$3000-3200 \mathrm{~m}$} & & & $\mathrm{C}$ \\
\hline \multicolumn{3}{|c|}{ Subpáramo (Páramo bajo) } & \multicolumn{2}{|c|}{$3200-3500(-3600) \mathrm{m}$} & & & $\mathrm{C}$ \\
\hline \multicolumn{3}{|c|}{ Páramo propiamente dicho } & \multicolumn{2}{|c|}{$(3500-) 3600-4100 \mathrm{~m}$} & & & $\mathrm{C}$ \\
\hline \multicolumn{3}{|c|}{ Superpáramo } & \multicolumn{4}{|c|}{$4100 \mathrm{~m}$ al límite inferior de las nieves perpetuas } & $\mathrm{C}$ \\
\hline \multicolumn{3}{|l|}{ Tropical } & \multicolumn{2}{|c|}{$0-1100 \mathrm{~m}$} & & & $\mathrm{D}$ \\
\hline \multicolumn{3}{|l|}{ Subandino } & \multicolumn{2}{|c|}{$1100-2350 \mathrm{~m}$} & & & $\mathrm{D}$ \\
\hline \multicolumn{3}{|l|}{ Andino } & \multicolumn{2}{|c|}{$2350-3000 \mathrm{~m}$} & & & $\mathrm{D}$ \\
\hline \multicolumn{3}{|l|}{ Altoadino } & \multicolumn{2}{|c|}{$3000-3200 \mathrm{~m}$} & & & $\mathrm{D}$ \\
\hline \multicolumn{3}{|l|}{ Subpáramo } & \multicolumn{2}{|c|}{$3200-3500 \mathrm{~m}$} & & & $\mathrm{D}$ \\
\hline \multicolumn{3}{|l|}{ Páramo } & \multicolumn{2}{|c|}{$3500-4100 \mathrm{~m}$} & & & $\mathrm{D}$ \\
\hline \multicolumn{3}{|c|}{ Superpáramo } & \multicolumn{2}{|c|}{$>4100 \mathrm{~m}$} & & & $\mathrm{D}$ \\
\hline
\end{tabular}

troncos caídos sin corteza), hojarasca, roca, suelo y briófitos.

Cada muestra recibió un valor de $\begin{array}{llll}0 & 0 & 1\end{array}$ según su ausencia o presencia en una de las cordilleras y sus vertientes, los pisos altitudinales según sistema de clasificación, y según luz relativa y tipo de sustrato. Posteriormente, se combinaron los datos para todas las muestras de una especie para obtener un puntaje a nivel de especie y de esta manera también balancear datos inexistentes a nivel de muestra. Además de especies, también se separaron fotomorfos en especies fotosimbiodémicas (que producen talos diferentes con cianobacterias o algas verdes) y formas reproductivas en especies que tienen talos con apotecios (sexuales) o isidios (vegetativos). Esta clasificación se basó en resultados de un estudio filogenético molecular (Moncada, 2012; Moncada et al., 2013). Se obtuvo un total de 103 especies y siete biotipos adicionales y un total de 34 variables binarios: diez variables representando las cordilleras y sus vertientes, cuatro variables de los pisos altitudinales según Rangel-Ch. (1995, 2000), cuatro variables de los pisos altitudinales según Rodríguez et al. (2006b), siete variables de los pisos altitudinales según la clasificación combinada, tres variables de la luz relativa y seis variables del tipo de sustrato (Apéndice, versión digital).

Los datos obtenidos fueron sometidos a un análisis de ordenación de escalamiento multidimensional no métrico (NMS). Para ambos, se usó el coeficiente de correlación como índice de distancia y en ambos casos, se implementó una prueba al azar de randomización para analizar si los ejes principales eran significativamente diferentes de una configuración aleatoria ( $\mathrm{n}=249$ repeticiones). Los análisis fueron hechos con el programa PC-ORD $5.03(\mathrm{McCu}-$ ne \& Mefford, 1999; McCune \& Grace, 2002).

Adicionalmente, para cada especie, se calculó un valor ponderado para los diferentes sistemas de clasificación de pisos altitudinales 
y se computó la correlación lineal no paramétrica de Spearman para obtener una medida de consistencia entre los sistemas de clasificación ecogeográfica usados; esto fue realizado en el progama STATISTICA ${ }^{\mathrm{TM}} 6.0$.

\section{RESULTADOS}

Ecogeografía: De 1765 ejemplares revisados, se obtuvo un total de 103 especies y 7 biotipos adicionales, distribuídas principalmente en la Región Andina y la Región del Chocó Biogeográfico. De las 103 especies, 93 fueron únicas para la Región Andina, una para la Región del Chocó Biogeográfico $(S$. aff. venosa) y nueve se encontraron en las dos regiones ( $S$. dioica, $S$. impressula, $S$. minutula, S. papillata, S. pseudolimbata, S. roseocyphellata, S. scabrosa, S. subfilicinella, S. tomento$s a$ ). Siguiendo la clasificación de las unidades de paisaje con base en la topografía para la región del Chocó Biogeográfico Colombiano, propuesto por Poveda et al. (2004), las diez especies de Sticta pertenecientes a esta región se ubicaron todas en la Unidad de Paisaje Subandino Cordillerano.

Con base en la distribución del territorio Colombiano en zonas de vida propuestas por Rangel-Ch. (1995), la distribución de las especies de Sticta mostró una clara preferencia por la Región Andina, con pocas especies en la Región Septentrional (Apéndice, versión digital). Dentro de esta región, las zonas de vida Subandina, Andina y Páramo fueron las que mostraron mayor diversidad de especies. En la zona de vida Subandina, el total de especies encontradas fue 56, en la zona de vida Andina 83 y en el Páramo 52, indicando que la zona de vida Andina correspondería al centro de dispersión para numerosas especies, que luego se extienden a las zonas de vidas Subandina y Páramo y, en algunos casos ( $S$. andreana, $S$. hypoglabra, S. isidiodichotoma, S. laselvae, $S$. scabrosa, $S$. aff. weigelii), hasta la zona de vida Tropical.

Utilizando la clasificación de pisos bioclimáticos (Rodríguez et al., 2006b), se obtuvo un resultado semejante, con excepción de algunas especies que cambian de zona de vida debido a su ubicación en las tres cordilleras principales (Apéndice, versión digital). Estas especies incluyen a Sticta neolinearis, la cual clasifica como andina en el sistema de Rangel-Ch. (1995) y andino-subparamuna en el sistema de Rodríguez et al. (2006b), S. sylvatica (andinaparamuna vs. subandina-paramuna), S. silverstonii (subandina vs. andina), la forma isidiada de $S$. dioica (subandina-andina vs. andina), $S$. leucoblepharis, S. lineariloba y S. paramuna (subandino-paramunas vs. andinas), y el cloromorfo de $S$. macrothallina, el cianomorfo de $S$. neopulmonarioides, y $S$. subfilicinella (subandino-paramunas vs. subandino-andinas).

Usando la combinación de Rangel-Ch. (1995, 2000) y Rodríguez et al. (2006b), y denominando cada una de las subdivisiones como ecozonas (Cuadro 1), las ecozonas con mayor diversidad de especies de Sticta son nuevamente los pisos subandino a páramo (Apéndice, versión digital). Al comparar la distribución de especies de Sticta según el sistema de clasificación utlizado, existe una fuerte correlación entre los valores basados en los dos sistemas, con $\mathrm{r}_{\text {Spearman }}=0.92(\mathrm{p}<0.001)$. El sistema combinado muestra correlaciones menos fuertes pero también altamente significativas con cada una de las clasificaciones, con $\mathrm{r}_{\text {Spearman }}=0.83(\mathrm{p}<0.01)$ tanto con el sistema de Rangel-Ch. (1995) como con el de Rodríguez et al. (2006b).

Utilizando la división de las cordilleras principales, se observó que un gran número de unidades taxonómicas de Sticta están distribuidas en dos o tres de las cordilleras principales (Apéndice, versión digital). Un número menor de especies parecen restringidas a una de las tres cordilleras, teniendo la Cordillera Oriental el más alto número de especies exclusivas. No existe una diferencia marcada en el número total de unidades taxonómicas encontradas en cada una de las cordilleras, con 76 en la Cordillera Occidental, 94 en la Cordillera Central y 80 en la Cordillera Oriental. Existe una ligera diferencia en la diversidad de especies comparando las vertientes orientales y occidentales de las cordilleras, siendo las vertientes orientales 
las que tienen un número ligeramente mayor, con excepción de la Cordillera Central (Apéndice, versión digital).

Microhabitat y sustrato: Usando la clasificación de luz relativa en tres categorías (sombreada, intermedia, expuesta), las especies de Sticta se pueden dividir en grupos que toleran un alto rango de intensidad lumínica contrastando con las que tienen un rango más estrecho (Apéndice, versión digital). Se observa que especies con fotobionte cianobacterial tienden a tolerar intensidades lumínicas más altas, mientras que las especies que tienen fotobionte verde tienden a encontrarse en microhabitats sombreados a moderadamente expuestos.

El sustrato preferido por la mayoría de las especies de Sticta es la corteza de árboles y arbustos (Apéndice, versión digital). Sin embargo, numerosas especies suelen ser generalistas, o también encontrándose sobre rocas o suelo, frecuentemente mezcladas con briófitos.
Algunas especies suelen tener preferencias pronunciadas por un tipo de sustrato creciendo casi exclusivamente sobre corteza, rocas o suelo, como $S$. andensis, $S$. arbuscula, $S$. cometia, $S$. fuliginosa, S. globulifuliginosa, S. gyalocarpa, S. isidiokunthii, S. laselvae, S. lobarioides, $S$. luteocyphellata, S. macrofuliginosa, $S$. macrothallina, S. microisidiata, S. minutula, S. papillata, S. paramuna, S. pulmonarioides, $S$. robertiana, S. rubropruinosa, S. rudiuscula, S. silverstonii, S. subfilicinella, S. tatamana y $S$. beauvoisii.

Diferenciación ecogeográfica de biotipos: En las especies que presentan dos biotipos diferentes, una con reproduccion sexual vs. otra con reproducción vegetativa o representando diferentes fotomorfos de una especie fotosimbiodémica, se puede observar diferencias ecogeográficas entre ellas. En Sticta dioica, la forma isidiada presenta un rango más estrecho que la forma sexual, tanto en términos de

\section{CUADRO 2}

Especies de Sticta con modo de reproducción y fotosimbiodemas diferenciadas en el territorio colombiano

\begin{tabular}{|c|c|c|c|c|c|c|c|}
\hline Especie & Forma & Altitud & Piso & Luz relativa & Cordillera-Vertiente & Sustrato & $\begin{array}{l}\text { Distribución } \\
\text { geopolítica }\end{array}$ \\
\hline \multirow[t]{2}{*}{ dioica } & isidiada & $2450-3220 \mathrm{~m}$ & Suband-andino & $\begin{array}{l}\text { Sombr.- } \\
\text { Intermedia }\end{array}$ & Orie & Corteza & Huila, Risaralda \\
\hline & sexual & $1700-3600 \mathrm{~m}$ & Suband-param & $\begin{array}{l}\text { Sombr.- } \\
\text { Intermedia }\end{array}$ & Cent, Orie-Occi & Corteza & amplia \\
\hline \multirow[t]{2}{*}{ lobarioides } & ciano & $2400 \mathrm{~m}$ & Suband & Media & $\begin{array}{l}\text { Cent-Orie, } \\
\text { Orie-Orie }\end{array}$ & Corteza & Huila, Risaralda \\
\hline & cloro & $1450-3500 \mathrm{~m}$ & Suband-param & $\begin{array}{l}\text { Sombr.- } \\
\text { Intermedia }\end{array}$ & todas & Corteza & amplia \\
\hline \multirow[t]{2}{*}{ macrothallina } & ciano & $2600 \mathrm{~m}$ & Andino & Intermedia & Occi-Orie & Corteza & Risaralda \\
\hline & cloro & $1800-3000 \mathrm{~m}$ & Andino & Intermedia & $\begin{array}{l}\text { Cent-Orie, } \\
\text { Orie-Orie }\end{array}$ & Corteza & $\begin{array}{l}\text { Magdalena, Risa- } \\
\text { ralda, Tolima }\end{array}$ \\
\hline \multirow[t]{2}{*}{ neopulmonarioides } & ciano & $2000-3460 \mathrm{~m}$ & Altoandino & Intermedia & todas & Corteza, Roca & amplia \\
\hline & cloro & $2400-3500 \mathrm{~m}$ & Andino-param & Intermedia & $\begin{array}{l}\text { Cent-Orie, } \\
\text { Orie-Orie }\end{array}$ & $\begin{array}{l}\text { Corteza, } \\
\text { Madera }\end{array}$ & $\begin{array}{l}\text { Magdalena, Nariño, } \\
\text { Risaralda, Tolima }\end{array}$ \\
\hline \multirow[t]{2}{*}{ phyllidiokunthii } & ciano & $1400-3930 \mathrm{~m}$ & Suband-param & $\begin{array}{l}\text { Intermedia - } \\
\text { Expuesta }\end{array}$ & Cent, Orie & Corteza & amplia \\
\hline & cloro & $1860-2600 \mathrm{~m}$ & Suband-andino & Intermedia & $\begin{array}{l}\text { Cent-Orie, } \\
\text { Orie-Orie }\end{array}$ & $\begin{array}{l}\text { Corteza, } \\
\text { Madera }\end{array}$ & Huila, Risaralda \\
\hline \multirow[t]{2}{*}{ pseudolobaria } & ciano & $2400 \mathrm{~m}$ & Andino & Intermedia & Occi-Orie & Corteza & Risaralda \\
\hline & cloro & $2100-3750 \mathrm{~m}$ & Andino & $\begin{array}{l}\text { Sombr.- } \\
\text { Intermedia }\end{array}$ & todas & $\begin{array}{l}\text { Corteza, } \\
\text { Madera, Roca }\end{array}$ & amplia \\
\hline \multirow[t]{2}{*}{ tatamana } & ciano & $2800 \mathrm{~m}$ & Andino & Intermedia & Occi-Orie & Corteza & Risaralda \\
\hline & cloro & $2800 \mathrm{~m}$ & Andino & Intermedia & Occi-Orie & Corteza & Risaralda \\
\hline
\end{tabular}


zonas de vida, número de cordilleras y sustrato (Cuadro 2). Diferencias semejantes también se aprecian para los diferentes fotomorfos de las especies fotosimbiodémicas.

La ordenación por escalamiento multidimensional no métrico (NMS) dió un resultado con configuración significativa (Fig. 1) para tres ejes extraídos (estrés final medio $=18.95$; estrés final medio randomizado $=27.63 ; \mathrm{p}<0.01$; estrés final medio para la solución tridimensional=17.45). Muestra una agrupación principal de especies según su distribución entre las cordilleras y una fuerte correlación con los pisos bioclimáticos combinados de las clasificaciones de Rangel-Ch. $(1995,2000)$ y Rodríguez et al. (2006a). Es notable que las especies que son exclusivas para la Cordillera Central se localizan centralmente en el diagrama de ordenación, igual como las especies que se encuentran en dos o tres de las cordilleras principales, indicando que la Cordillera Central es el centro de distribución para el género Sticta. Los resultados también mostraron que las especies no tienen distribuciones ecogeográficas aleatorias sino que por el contrario, forman grupos distintos de especies ecogeográficamente equivalentes.

\section{DISCUSIÓN}

El presente estudio demuestra que las especies de Sticta en el territorio colombiano no se distribuyen de forma aleatoria sino que tienen patrones de distribución marcados con relación a las diferentes zonas de vida. RangelCh. (2000) menciona que la separación entre la franja de subpáramo y páramo propiamente dicho no es muy clara y que depende, además

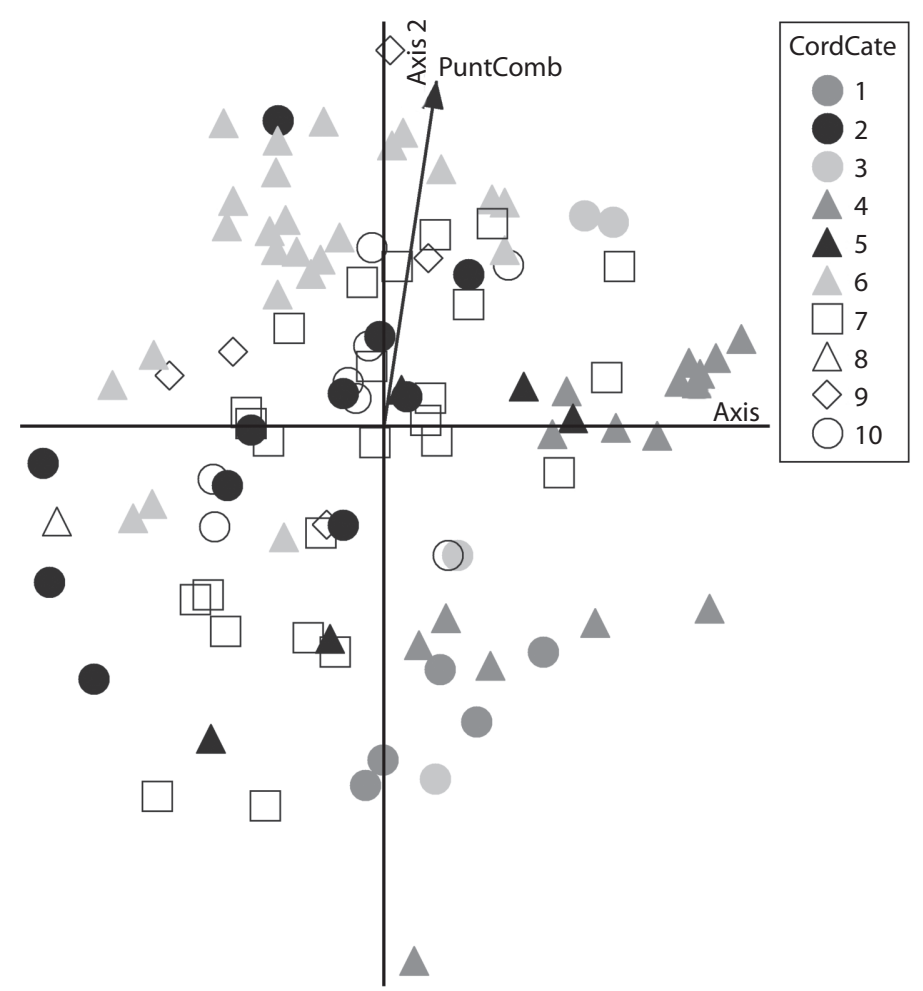

Fig. 1. Ordenación de especies de Sticta según puntajes por variables ambientales usando escalamiento multidimensional no métrico (NMS). Los símbolos indican su distribución entre las cordilleras principales $(1=$ Occidental, $2=$ Central, 3 = Oriental, $4=$ Occidental-Central, $5=$ Occidental-Oriental, $6=$ Central-Oriental, $7=$ Occidental-Central-Oriental, $8=$ Oriental-Septentrional, 9 = Central-Oriental-Septentrional, 10 = todas las cordilleras). La flecha indica la correlación de los ejes principales con el puntaje combinado (medio ponderado) de pisos bioclimáticos. 
de la altitud, del factor fisiográfico-topográfico. Con relación a eso, Rodríguez et al. (2006b) argumentan que, dependiendo del flanco de cada una de las cordilleras, las fronteras de los pisos bioclimáticos pueden variar. Sin embargo, es aparente que el uso de diferentes sistemas de clasificación ecogeográfica, y la combinación entre ellos implementada aquí, no afectan significativamente la caracterización de las especies de líquenes, ya que en todas las clasificaciones, las zonas de vida subandinas a paramunas son las que tienen la mayor diversidad de especies.

Esto confirma al género Sticta como un grupo principalmente de zonas de clima frío a templado que en las latitudes tropicales está concentrado en las zonas altas, por encima de los 2000m (Galloway, 1994, 1997, 1998, 2001, 2007), especialmente en aquellas áreas de vegetación boscosa y arbustiva subandina, andina y paramuna donde, probablemente la elevada y constante humedad y la temperatura no mayor a $20^{\circ} \mathrm{C}$, permiten el establecimiento y supervivencia de estos líquenes. Por lo tanto, se puede asumir que la orogenia andina debe haber tenido una influencia marcada en la especiación de este género en el Neotrópico y particularmente en el norte de Suramérica, donde los Andes se dividen en tres cordilleras principales. La observación que la Cordillera Oriental forma un centro de distribución para las especies de Sticta en Colombia sugiere que la diversificación del género ocurrió a partir de esta Cordillera, que forma la prolongación más antigua de los Andes llegando del sur de Suramérica (Hoorn et al., 2010) hasta Venezuela. Un estudio usando un reloj molecular estimó la edad del nodo basal de Sticta en 26 millones de años (Lücking, 2012), lo que coincide con el período de mayor levantamiento de los Andes del norte de Suramérica, desde hace 25 millones de años, con un período más intenso para alcanzar altitudes de encima de $3000 \mathrm{~m}$ entre 10 y 5 millones de años atrás (Hoorn et al., 2010).

Considerando la intensidad lumínica, se puede reconocer que las especies con cianobacterias como fotobionte primario son frecuentes en todos los lugares, desde sombreados a expuestos, probablemente por la capacidad de absorber agua líquida que poseen las cianobacterias, además de la fijación de nitrógeno (Kelly \& Becker, 1975; Becker, 1980; Green et al., 1980, 1985; Guzmán et al., 1990; Green \& Lange, 1991; Beckett, 1995; Zotz et al., 1998; Antoine, 2004; Benner et al., 2007). También podría deberse a las diferentes adaptaciones cromáticas de las cianobacterias en la utilización de numerosos pigmentos, entre ellos los ficobilínicos, que les permiten absorber energía lumínica hasta a bajas intensidades de luz (Grossman, Schaefer, Chiang \& Collier, 1993; Kehoe, 2010). Al contrario, las especies con fotobionte primario verde son más frecuentes en lugares sombreados a intermedios, con frecuencia en ramas secundarias de árboles y arbustos de bosques subandino y andinos, donde estas ramas y los briófitos asociados pueden protegerlos de la excesiva evaporación del agua.

El análisis cuantitativo de los datos usando técnicas de ordenación señala que el factor dominante para la formación de grupos de especies ecogeográficamente equivalentes es el gradiente altitudinal, lo que coincide con las zonas de vida clasificadas como tropical, subandina, andina y paramuna, además de las subzonas de vida altoandina, subpáramo y superpáramo. El segundo factor importante es la división geográfica entre las tres cordilleras y sus vertientes, acompañado por ligeras diferencias en las preferencias altitudinales. Esto coincide con la idea que el efecto de altitud es ligeramente diferente según cada vertiente, porque la dirección prevalente de los vientos causa que zonas comparables en temperatura y precipitación se ubiquen en altitudes ligeramente diferentes comparando las vertientes occidentales y orientales (Rodríguez et al., 2006b).

\section{AGRADECIMIENTOS}

Los autores deseamos agradeceder a todas las personas que contribuyeron en el desarrollo de este estudio. En especial a la Universidad Distrital Francisco José de Caldas, por el apoyo 
económico, académico y logístico, a la Universidad Nacional de Colombia, al Field Museum Chicago y a la NSF por su apoyo dentro del proyecto Neotropical Epiphytic Microlichens - An Innovative Inventory of a Highly Diverse yet Little Known Group of Symbiotic Organisms (DEB 715660 to The Field Museum; PI R. Lücking). A los curadores de los herbarios nacionales ANDES, COAH, COL, CUVC, FAUC. HUA, UDBC y UPTC; e internacionales B, BM, E, F, G, H, L, MEL, OXF, PC, TUR, U, US y W, especialmente Carlos Parra (COL), Harrie Sipman (B), Holger Thüs (BM), Bruno Dennetiére (PC) y Thorsten Lumbsch (F) por su valioso tiempo y colaboración durante las visitas a esos herbarios y por el envío de ejemplares Tipo y colecciones históricas que fueron determinantes para esta investigación. A Alejandra Suárez por su invaluable trabajo de campo, laboratorio y captura de datos. A los integrantes del Grupo Colombiano de Liquenología por su colaboración en campo y el envío de material, en especial a Luisa Betancourt, Lady Vargas, Leidy Herrera, Isabel Ardila, Luis Fernando Coca, Edier Soto, David Diaz y Diego Simijaca.

\section{RESUMEN}

Colombia es un país megadiverso, pero con una ausencia significativa en el conocimiento de la taxonomía y ecología de hongos y líquenes. Por lo tanto, el objetivo del presente estudio era un análisis ecogeográfico de las especies de Sticta de Colombia. Los datos incluyeron la macrodistribución (con respeto a las cordilleras y zonas de vida) y preferencias de microhabitat (luz, sustrato) y fueron obtenidos mediante trabajo de campo y en el herbario (complemetados con mapas modernos de geografía y vegetación), para 103 especies actualmente reconocidas en Colombia (más siete biotipos con diferentes fotobiontes o modo de reproducción). Se elaboró un análisis de ordenación usando la técnica de escalamiento multidimensional no métrico (NMS), para establecer correlaciones entre variables ecogeográficas y las especies y para definir ecotipos. Las especies de Sticta en Colombia presentan patrones de distribución relativos a la altitud, las zonas de vida y la ubicación en las cordilleras, tanto como luz y sustrato, formando varios grupos distintos. Las especies con cianobionte tienen un rango de distribución más amplio que las especies con fotobionte verde. Concluimos que la orogenia de los Andes afectó significativamente la especiación del género Sticta y su diferenciación ecogeográfica.
Palabras clave: Sticta, zonas de vida, patrones de distribución, subandino, andino, páramo, superpáramo.

\section{REFERENCIAS}

Aguirre-C., J. A., \& Sipman, H. J. M. (2004). Diversidad y riqueza de líquenes en el Chocó Biogeográfico. In J. O. Rangel-Ch (Ed.), Colombia Diversidad Biótica IV. El Chocó Biogeográfico / Costa Pacífica (pp. 455474). Bogotá: Universidad Nacional de Colombia \& Conservación Internacional.

Aguirre-C., J. A., \& Rangel-Ch., J. O. (2008a). Riqueza y aspectos ecológicos y fitogeográficos sobre la flora de líquenes. In J. O. Rangel-Ch. (Ed.), Colombia Diversidad Biótica VI. Riqueza y diversidad de los musgos y líquenes de Colombia (pp. 549-559). Bogotá: Instituto de Ciencias Naturales, Universidad Nacional de Colombia.

Aguirre-C., J. A., \& Rangel-Ch., J. O. (2008b). Riqueza y aspectos eco y fitogeográficos sobre la flora de musgos. In J. O. Rangel-Ch. (Ed.), Colombia Diversidad Biótica VI. Riqueza y diversidad de los musgos y líquenes de Colombia (pp. 321-335). Bogotá: Instituto de Ciencias Naturales, Universidad Nacional de Colombia.

Álvarez, E. (2001). Diseño de inventarios en biodiversidad: métodos no paramétricos para la estimación de la riqueza de especies y la complementariedad biótica. Ibagué. Interconexión Eléctrica S.A. (ISA).

Amat, G. G., Andrade-C., M. G., \& Amat, E. C. (Eds.). (2007). Libro Rojo de invertebrados terrestres de Colombia. Serie Libros Rojos de especies amenazadas de Colombia. Bogotá, Colombia. Conservación Internacional Colombia, Instituto de Ciencias Naturales Universidad Nacional de Colombia, Ministerio de Ambiente Vivienda y Desarrollo Territorial.

Andrade-C., M. G. (2011). Estado del conocimiento de la biodiversidad en Colombia y sus amenazas - consideraciones para fortalecer la interacción cienciapolítica. Revista Academia Colombiana de Ciencias Exactas Físicas y Naturales, 35, 492-508.

Antoine, M. E. (2004). An ecophysiological approach to quantifying nitrogen fixation by Lobaria oregana. Bryologist, 107, 82-87.

Aptroot, A., \& Iqbal, S. H. (2011). Some lichens of Bangladesh. Bryologist, 114, 466-468.

Barney-D.,V. E. (2011). Biodiversidad y ecogeografia del género Lupinus l. (Leguminosae) en Colombia (Tesis de Maestría). Universidad Nacional de Colombia, Palmira, Colombia.

Becker, V. E. (1980). Nitrogen fixing lichens in forests of the Southern Appalachian Mountains of North Carolina. Bryologist, 83, 29-39. 
Beckett, R. P. (1995). Some aspects of the water relations of lichens from habitats of contrasting water status studied using thermocouple psychrometry. Annals of Botany, 76, 211-217.

Benavides-Molineros, J., Castiblanco-A., J., \& VivasSegura, A. J. (2006). Biodiversidad en cifras.Tomo II. In M. E. Chaves \& M. Santamaría (Eds.), Informe sobre el avance en el conocimiento y la información de la biodiversidad 1998-2004 (pp. 23-39). Bogotá D.C. Colombia: Instituto de Investigación de Recursos Biológicos Alexander von Humboldt.

Benner, J. W., Conroy, S., Lunch, C. K., Toyoda, N., \& Vitousek, P. M. (2007). Phosphorus fertilization increases the abundance of nitrogenase activity of the cyanolichen Pseudocyphellaria crocata in Hawaiian montane forests. Biotropica, 39, 400-405.

Bernal, R., Celis, M., \& Gradstein, S. R. (2007). Plant diversity of Colombia catalogued. Taxon, 56, 273.

Boonpragop, K., \& Polyiam, W. (2007). Ecological groups of lichens along environmental gradients on two different host tree species in the tropical rain forest at Khao Yai National Park, Thailand. Bibliotheca Lichenologica, 96, 25-48.

Cáceres, M. E. S., Lücking, R., \& Rambold, G. (2007). Phorophyte specificity and environmental parameters as determinants for species composition, richness and area cover in corticolous crustose lichen communities in the Atlantic rainforest of northeastern Brazil. Mycological Progress, 6, 117-136.

Cáceres, M. E. S., Lücking, R., \& Rambold, G. (2008). Corticolous microlichens in northeastern Brazil: habitat differentiation between coastal Mata Atlântica, Caatinga and Brejos de Altitude. Bryologist, 111, 98-117.

Chaves, M. E., \& Santamaría, M. (Eds). (2006). Informe Nacional sobre el avance en el conocimiento y la información de la biodiversidad 1998-2004. Instituto de Investigación en Recursos Biológicos Alexander von Humboldt. 2 Tomos, Bogotá D.C. Colombia.

Crespo, P., Célleri, R., Buytaert, W., Feyen, J., Iñiguez, V., Borja, P., \& De Bièvre, B. (2010). Land use change impacts on the hydrology of wet Andean páramo ecocystems. In Status and Perspectives of Hydrology in Small Basins. IAHS Publ. 336.

Del Prado, R., Schmitt, I., Kautz, S., Palice, Z., Lücking, R., \& Lumbsch, H. T. (2006). Molecular data place Trypetheliaceae in Dothideomycetes. Mycological Research, 110, 511-520.

De Brievre, B., \& Calle, T. (2011). The Andean Paramo Project: Conserving biodiversity and hydrological services on the roof of the Andes. Mountain Forum Bulletin, 1-6.

Díaz, M., Solis, A. E., \& Brochero, H. L. (2011). Distribución geográfica de Neoleucinodes elegantalis
(Lepidoptera: Crambidae) en Colombia. Revista Colombiana de Entomología, 37, 71-76.

Dyer, L. A., \& Letourneau, D. K. (2007). Determinants of lichen diversity in a rain forest understory. Biotropica, 39, 525-529.

Feuerer, T., \& Hawksworth, D. L. (2007). Biodiversity of lichens, including a world-wide analysis of checklist data based on Takhtajan's floristic regions. Biodiversity and Conservation, 16, 85-98.

Galloway, D. J. (1979). Biogeographical elements in the New Zealand lichen flora. In D. Bramwell, (Ed.), Plants and Islands (pp. 201-224). Londres: Academic Press.

Galloway, D. J. (1994). Studies on the lichen genus Sticta (Schreber) Ach.: I. Southern South American species. The Lichenologist, 26, 223-282.

Galloway, D. J. (1997). Studies on the lichen genus Sticta (Schreber) Ach. IV. New Zealand species. The Lichenologist, 29, 105-168.

Galloway, D. J. (1998). Studies on the lichen genus Sticta (Schreber) Ach. V. Australian species. Tropical Bryology, 15, 117-160.

Galloway, D. J. (2001). Sticta. Flora of Australia. Volume 58a, Lichens, 3, 78-97.

Galloway, D. J. (2007). Flora of New Zealand Lichens (2nd ed.). Lincoln: Manaaki Whenua Press.

Green, T. G. A., \& Lange, O. L. (1991) Ecophysiological adaptations of the lichen genera Pseudocyphellaria and Sticta to south temperate rainforests. The Lichenologist, 23, 267-282.

Green, T. G. A., Horstmann, J., Bonnett, H., Wilkins, A., \& Silvester, W. B. (1980). Nitrogen fixation by members of the Stictaceae (Lichenes) of New Zealand. New Phytologist, 84, 339-348.

Green, T. G. A., Snelgar, W. P., \& Wilkins, A. L. (1985). Photosynthesis, water relations and thallus structure of Stictaceae lichens. In D. H. Brown (Ed.), Lichen physiology and cell biology (pp. 57-75). Nueva York \& Londres: Plenum Press.

Grossman, A. R., Schaefer, M. R., Chiang, G. G., \& Collier, J. L. (1993). The phycobilisome, a lightharvesting complex responsive to environmental conditions. Microbiological reviews, 57(3), 725-749.

Guzmán, G., Quilhot, W., \& Galloway, D. J. (1990). Decomposition of species of Pseudocyphellaria and Sticta in a southern Chilean forest. The Lichenologist, $22,325-331$.

Hawksworth, D. L. (1991). The fungal dimension of biodiversity: magnitude, significance, and conservation. Mycological Research, 95, 641-655.

Hawksworth, D. L. (2001). The magnitude of fungal diversity: the 1.5 million species estimate revisited. Mycological Research, 105, 1422-1432. 
Hernández, C. J., Walschburger, B. T., Ortiz, Q. R., \& Hurtado, G. A. (1992). Origen y distribución de la biota suramericana y colombiana. In G. Halffter (Ed.), La Diversidad Biológica de Iberoamérica. Acta Zoológica Mexicana (n.s.) (pp. 3-24). Volumen especial de 1992. CYTEDD, Programa Iberoamericano de Ciencia y Tecnología para el Desarrollo, México D.F.

Herrera-Campos, M. A., Lücking, R., Pérez, R.-E., Campos, A., Colín, P. M., \& Bárcenas-Peña, A. (2004). The foliicolous lichen flora of Mexico. V. Biogeographical affinities, altitudinal preferences, and an updated checklist of 293 species. The Lichenologist, 36, 309-327.

Holz, I., \& Gradstein, S. R. (2005). Cryptogamic epiphytes in primary and recovering upper montane oak forests of Costa Rica - Species richness, community composition and ecology. Plant Ecolology, 178, 89-109.

Hoorn, C., Wesselingh, F. P., ter Steege, H., Bermudez, M. A., Mora, A., Sevink, I., Sanmartín, J., SanchezMeseguer, A., Anderson, C. L., Figueiredo, J., Jaramillo-O., C., Riff, D., Negri, F. R., Hooghiemstra, H., Lundberg, J., Stadler, T., Sarkinen, T., \& Antonelli, A. (2010). Amazonia through time: Andean uplift, climate change, landscape evolution and biodiversity. Science, 330, 927-931.

Kelly, B. B. \& Becker, V. E. (1975). Effects of light intensity and temperature on nitrogen fixation by Lobaria pulmonaria, Sticta weigelii, Leptogium cyanescens and Collema subfurvum. Bryologist, 78, 350-355.

Kelly, D. L., O’Donovan, G., Feehan, J., Muphy, S., Drangeid, S. O., \& Marcano-Berti, L. (2004). The epiphyte communities of a montane rain forest in the Andes of Venezuela: patterns in the distribution of the flora. $J$. Tropical Ecology, 20, 643-666.

Kehoe, D. M. (2010). Chromatic adaptation and the evolution of light color sensing in cyanobacteria. Proceedings of the National Academy of Sciences, 107 (20), 9029-9030.

Komposch, H., \& Hafellner, J. (2000). Diversity and vertical distribution of lichens in a Venezuelan tropical lowland rain forest. Selbyana, 21, 11-24.

Komposch, H., \& Hafellner, J. (2002). Life form diversity of lichenized fungi in an Amazon lowland rainforest. Bibliotheca Lichenologica, 82, 311-326.

Komposch, H., \& Hafellner, J. (2003). Species composition of lichen dominated corticolous communities: a lowland rain forest canopy compared to an adjacent shrubland in Venezuela. Bibliotheca Lichenologica, 86, 351-367.

Lücking, R. (1998a). Ecology of foliicolous lichens at the "Botarrama" Trail (Costa Rica), a neotropical rainforest site. Part II. Patterns of diversity and area cover, and their dependence on microclimate and phorophyte species. Ecotropica, 4, 1-24.
Lücking, R. (1999a). Ecology of foliicolous lichens at the "Botarrama" Trail (Costa Rica), a neotropical rain forest. I. Species composition and its ecogeographical implications. Biotropica, 31, 553-564.

Lücking, R. (1999b). Ecology of foliicolous lichens at the 'Botarrama' Trail (Costa Rica), a neotropical rainforest. IV. Species associations, their salient features and their dependence on environmental variables. The Lichenologist, 31, 269-289.

Lücking, R. (1999c). Líquenes folícolas de la Estación Biológica La Selva, Costa Rica: Inventario, comunidades y comparación florística de tipos de vegetación. Revista de Biología Tropical, 47, 287-308.

Lücking, R. (2003). Takhtajan's floristic regions versus foliicolous lichen biogeography: a compatibility analysis. The Lichenologist, 35, 33-54.

Lücking, R. (2012). One species is many: unrecognized levels of diversification of tropical macrolichens in Neotropical paramos. 49th Annual Meeting of the Association for Tropical Biology and Conservation (ATBC 2012), "Ecology, evolution and sustainable use of tropical biodiversity", Bonito, Mato Grosso do Sul, Brazil, 13-22 June 2012. Abstracts: S03.OC.01.

Lücking, R., Rivas Plata, E., Chaves, J. L., Umaña, L., \& Sipman, H. J. M. (2009). How many tropical lichens are there... Really? Bibliotheca Lichenologica, 100, 399-418.

Luteyn, J. L. (1999). Páramos: A checklist of plant diversity, geographical distribution, and botanical literature. New York: The New York Botanical Garden Press.

Martins, S. M. A. (2006). Estudo da comunidade liquenizada epífita em Dodonaea viscosa L. na restinga do Parque Estadual de Itapuã, Viamão, R. S. (Ph.D. Thesis) Instituto de Botânica de São Paulo, Brazil.

McCune, B., \& Grace, J. B. (2002). Analysis of ecological communities. MjM Software, Gleneden Beach, Oregon.

McCune, B., \& Mefford, M. J. (1999). PC-ORD. Multivariate analysis of ecological data, Version 4.0. MjM Software, Gleneden Beach, Oregon, USA.

Molina, M. C., Crespo, A., Blanco, O., Lumbsch, H. T., \& Hawksworth, D. L. (2004). Phylogenetic relationships and species concepts in Parmelia s.str. (Parmeliaceae) inferred from nuclear ITS rDNA and b-tubulin sequences. The Lichenologist, 36, 37-54.

Moncada, B. (2012). El género Sticta en Colombia, Taxonomía, Ecogeografía e Importancia. (Tesis doctoral). Universidad Nacional de Colombia, Bogotá, Colombia.

Moncada, B., \& Lücking, R. (2012). Ten new species of Sticta and counting: Colombia as a hot spot for unrecognized diversification in a conspicuous macrolichen genus. Phytotaxa, 74, 1-29. 
Moncada, B., Lücking, R., \& Suárez, A. (2013). Molecular phylogeny of the genus Sticta (lichenized Ascomycota: Lobariaceae) in Colombia. Fungal Diversity (online first): DOI 10.1007/s13225-013-0230-0

Nash, T. H. III (Ed.). (2008). Lichen Biology. Cambridge: Cambridge University Press.

Nylander, W. (1863a). Lichenographiae Novo Granatensis Prodromus. Acta Societatis Scientiarum Fennicae, 7, 415-504.

Nylander, W. (1863b). Lichenes. In J. Triana, \& J. E. Planchon. Prodromus Florae Novo-Granatensis ou Énumération des plantes de la Nouvelle-Grénade avec description des espèces nouvelles. Annales des Sciences Naturelles Bot., Sér. 4, 19, 286-382.

Nylander, W. (1864). Circa Lichenum Novo a Granatensium novas explorations Lindigianas. Flora, 47, 617-619.

Nylander, W. (1867). Lichens Additamentum. Annales des Sciences Naturelles Botanique, Série, 5, 7, 536-537.

Poveda, I. C., Rojas, C. A., Rudas, P. A., \& Rangel-Ch., J. O. (2004). El Chocó biogeográfico: ambiente físico. In J. O. Rangel-Ch. (Ed.), Colombia Diversidad Biótica IV. El Chocó biogeográfico / Costa Pacífica (pp. 1-21). Bogotá D.C. Colombia: Instituto de Ciencias Naturales e Inderena.

Rangel-Ch., J. O. (Ed.). (1995). Colombia diversidad biótica I. Bogotá: Instituto de Ciencias Naturales e Inderena

Rangel-Ch., J. O. (2000). La Región Paramuna y franja aledaña en Colombia. In J. O. Rangel-Ch., (Ed.), Colombia Diversidad Biótica III. La Región de vida Paramuna (pp. 1-23). Bogotá: Instituto de Ciencias Naturales e Instituto Alexander von Humboldt.

Renjifo, L. M., Franco-Maya, A. M., Amaya-Espinel, J. D., Kattan, G. H., \& López-Lanús, B. (Eds.). (2002). Libro Rojo de las aves de Colombia. Serie Libros Rojos de especies amenazadas de Colombia. Bogotá: Colombia. Instituto Alexander von Humboldt \& Ministerio de Ambiente Vivienda y Desarrollo Territorial.

Rincón-Espitia, A., Aguirre-C. J., \& Lücking, R. (2011). Líquenes corticícolas en el caribe Colombiano. Caldasia, 33, 331-347.

Rodríguez-M., J. V., Alberico, M., Trujillo, F., \& Jorgenson, L. (Eds.). (2006a). Libro Rojo de los mamíferos de Colombia. Serie Libros Rojos de especies amenazadas de Colombia. Bogotá, Colombia: Conservación Internacional Colombia \& Ministerio de Ambiente Vivienda y Desarrollo Territorial.

Rodríguez, N., Armenteras, D., Morales, M., \& Romero, M. (2006b). Ecosistemas de los Andes Colombianos, segunda edición. Bogotá, Colombia: Instituto de Investigación de Recursos Biológicos Alexander von Humboldt.
Samper, C., \& García, H. (Eds.). (2001). Estrategia nacional para la conservación de plantas. Bogotá, Instituto de Investigación de Recursos Biológicos Alexander von Humboldt.

Simijaca, D. F. (2011). Líquenes epifitos de Quercus humboldtii en el Parque Natural Municipal robledales de Tipacoque (Boyacá - Colombia), (Trabajo de Grado). Universidad Pedagógica y Tecnológica de Colombia, Sede Tunja, Colombia.

Sipman, H. J. M. (1984). Lichens of the Buritaca-La Cumbre Transect Sierra Nevada de Santa Marta, Colombia. Studies on Tropical Andean Ecosystems, 2, 185-188

Sipman, H. J. M. (1989). Lichen zonation in the Parque Los Nevados transect. Studies on Tropical Andean Ecosystems, 3, 461-483.

Sipman, H. J. M. (1992). The origin of the lichen flora of the Colombian páramos. In H. Balslev, \& J. L. Luteyn (Eds.), Páramo. An Andean Ecosystem under human influence (pp. 95-109). Londres: Academic Press.

Sipman, H. J. M. (1995). Preliminary review of the lichen biodiversity of the Colombian montane forests. In $\mathrm{S}$. P. Churchill, H. Balslev, E. Forero, \& J. L. Luteyn (Eds.), Biodiversity and Conservation of Neotropical Montane Forests (pp. 313-320). Bronx, Nueva York: New York Botanical Garden.

Sipman, H. J. M. (2006). Diversity and biogeography of lichens in Neotropical montane oak forests. In M. Kappelle (Ed.), Ecology and Conservation of Neotropical montane oak forests (pp. 69-81). Ecological Studies 185. Berlin and Heidelberg: Springer-Verlag.

Sipman, H. J. M., \& Cleef, A. M. (1979). Studies on Colombian cryptogams V. Taxonomy, distribution and ecology of macrolichens of the Colombian páramos: 1. Cladonia subgenus Cladina. Procceding Koninklijke Nederlandese Akademie Wetenschappen Series C 82, 223-241.

Sipman, H. J. M., Hekking, W., \& Aguirre-C., J. (2008). Checklist of lichenized and lichenicolous fungi from Colombia. Biblioteca José Jerónimo Triana No. 20. Bogotá: Instituto de Ciencias Naturales, Facultad de Ciencias, Universidad Nacional de Colombia.

Soto-M., E., \& Bolaños, A. C. (2010). Diversidad de líquenes cortícolas en el bosque subandino de la finca Zíngara (Cali, Valle del Cauca). Revista de Ciencias, 14, 35-44.

Soto-M., E., Lücking, R., \& Bolaños, A.-C. (2012). Especificidad de forófito y preferencias microambientales de los líquenes cortícolas en cinco forófitos del bosque premontano de finca Zíngara, Cali, Colombia. Revista de Biología Tropical, 60, 843-856.

Stearn, W. (1968). Humboldt, Bonpland, Kunth and tropical American botany. A miscellany on the nova genera et species plantarun. Verlag von J. Cramer. 
Tehler, A., Irestedt, M., Wedin, M., \& Ertz, D. (2010). The old world Roccella species outside Europe and macaronesia: taxonomy, evolution and phylogeny. Systematic Biodiversity, 8, 223-246.

Wolf, J. H. D. (1993a). Epiphyte communities of tropical montane rain forests in the northern Andes. I: Lower montane communities. Phytocoenologia, 22, 1-52.
Wolf, J. H. D. (1993b). Diversity patterns and biomass of epiphytic bryophytes and lichens along an altitudinal gradient in the Northern Andes. Annals of the Missouri Botanical Garden, 80, 928-960.

Zotz, G., Büdel, B., Meyer, A., Zellner, H., \& Lange, O. L. (1998). In situ studies of water relations and $\mathrm{CO}_{2}$ exchange of the tropical macrolichen, Sticta tomentosa. New Phytologist, 139, 525-535. 


\section{APÉNDICE}

Distribución ecogeográfica y microhabitats preferidos de las especies y unidades taxonómicas del género Sticta en Colombia

\begin{tabular}{|c|c|c|c|c|c|c|}
\hline Especie & Cordilleras & Rang 1995 & Rodr 2006 & Combinación & Iluminación & Sustrato \\
\hline albocyphellata & Cent-Orie & Suba-Pára & Suba-Pára & Suba-Pára & Som-Exp & $\mathrm{Co}-\mathrm{Su}$ \\
\hline andensis & Occi-Cent & Andi-Pára & Andi-Pára & Alto-Pára & Inter & Co \\
\hline andina & todas & Suba-Pára & Suba-Pára & Suba-Supe & Som-Exp & Co-Ma-Ho-Ro-Su \\
\hline andreana & Orie & Trop-Andi & Basa-Andi & Trop-Andi & Som-Inter & Co-Ro \\
\hline arachnofuliginosa & Occi-Cent & Suba-Pára & Suba-Pára & Suba-Pára & Som-Exp & $\mathrm{Co}-\mathrm{Su}$ \\
\hline arachnosylvatica & Orie & Andi-Pára & Andi-Pára & Andi-Pára & Inter-Exp & Co \\
\hline arbuscula & Cent-Orie & Andi-Pára & Andi-Pára & Alto-Pára & Som-Exp & Co \\
\hline arbusculotomentosa & Cent-Orie-Sept & Andi-Pára & Andi-Pára & Andi-Pára & Inter & Co \\
\hline arcabucana & Orie & Andi & Andi & Alto-Subp & Inter & Co \\
\hline atroandensis & Orie & Pára & Pára & Pára-Supe & Inter-Exp & $\mathrm{Co}-\mathrm{Su}$ \\
\hline beauvoisii & Orie & Andi & Andi & Andi & Inter & $\mathrm{Su}$ \\
\hline brevior & Cent-Orie & Suba-Pára & Andi-Pára & Suba-Pára & Inter & $\mathrm{Co}-\mathrm{Su}$ \\
\hline caliginosa (aff.) & Orie & Andi & Andi & Andi & Inter & Co \\
\hline colombiana & Occi-Cent-Orie & Suba-Pára & Suba-Pára & Suba-Pára & Som-Exp & Co-Ma-Ro-Su-Br \\
\hline cometia & Occi-Cent-Orie & Suba-Pára & Suba-Pára & Suba-Pára & Inter & Co \\
\hline cometiella & Occi-Cent-Orie & Suba-Andi & Suba-Andi & Suba-Andi & Som-Inter & Co-Ma-Su \\
\hline cordillerana & Occi-Cent-Sept & Suba-Pára & Suba-Pára & Suba-Subp & Som-Inter & Co-Ho-Ro \\
\hline cordillerana (aff.) & Orie & Suba & Suba & Suba & Inter & $\mathrm{Co}$ \\
\hline cyphellulata (aff.) & Occi-Cent & Suba-Andi & Suba-Andi & Suba-Andi & Som-Inter & $\mathrm{Co}-\mathrm{Ma}$ \\
\hline dilatata & todas & Suba-Pára & Suba-Pára & Suba-Pára & Som-Inter & $\mathrm{Co}-\mathrm{Ma}$ \\
\hline dioica (isidiado) & Orie & Suba-Andi & Andi & Andi-Subp & Som-Inter & Co \\
\hline dioica (sexual) & Occi-Cent & Suba-Pára & Suba-Pára & Suba-Pára & Som-Inter & Co-Ho-Ro \\
\hline fuliginosa & Cent-Orie & Andi & Andi & Suba & Inter-Exp & Co \\
\hline fuscotomentosa & Occi-Cent & Suba-Andi & Suba-Andi & Suba-Alto & Som-Inter & Co \\
\hline gallowayana & Cent-Orie & Suba-Pára & Andi-Pára & Suba-Supe & Inter-Exp & Co-Ma-Ro-Su \\
\hline globulifuliginosa & Occi-Cent & Andi-Pára & Andi-Pára & Andi-Pára & Inter-Exp & Co \\
\hline granatensis & todas & Suba-Pára & Suba-Pára & Suba-Supe & Som-Exp & Co-Ma-Ro-Su \\
\hline gyalocarpa & Cent-Orie & Andi-Pára & Andi-Pára & Andi-Pára & Som-Exp & Co \\
\hline hirsutofuliginosa & Cent-Orie & Andi-Pára & Andi-Pára & Andi-Supe & Som-Exp & Co-Ro \\
\hline hirsutogyalocarpa & Cent-Orie & Andi-Pára & Andi-Pára & Alto-Supe & Som-Inter & $\mathrm{Co}-\mathrm{Su}$ \\
\hline hirta & Cent-Orie & Suba-Pára & Suba-Pára & Suba-Pára & Som-Inter & Co \\
\hline humboldtii & Cent-Orie & Suba-Pára & Suba-Pára & Suba-Pára & Som-Exp & Co-Ma-Su \\
\hline hypoglabra & Occi-Cent-Orie & Trop-Andi & Basa-Andi & Trop-Andi & Som-Exp & Co-Ro \\
\hline impressula & Occi-Cent-Orie & Suba-Pára & Suba-Pára & Suba-Pára & Som-Exp & todos \\
\hline isidiodichotoma & Cent & Trop & Basa & Trop & Inter & Co \\
\hline isidioimpressula & Occi-Cent & Andi-Pára & Andi-Pára & Andi-Pára & Inter-Exp & Co-Ro \\
\hline isidiokunthii & Cent-Orie & Suba-Pára & Andi-Pára & Suba-Pára & Inter-Exp & Co \\
\hline jaguirreana & Occi-Cent & Andi & Andi & Andi-Alto & Som-Exp & Co-Ma-Ro-Su \\
\hline laciniata (cf.) & Occi-Cent-Orie & Suba-Andi & Suba-Andi & Suba-Alto & Som-Inter & Co-Ma-Ho-Su \\
\hline laciniosa & Cent-Orie & Suba-Andi & Suba-Andi & Suba-Andi & Som-Inter & $\mathrm{Co}-\mathrm{Ma}$ \\
\hline laevis & Cent-Orie & Suba-Pára & Andi-Pára & Suba-Supe & Som-Inter & $\mathrm{Co}-\mathrm{Ro}-\mathrm{Su}-\mathrm{Br}$ \\
\hline laminobeauvoisii & Occi-Cent-Orie & Suba-Andi & Suba-Andi & Suba-Andi & Inter & Co-Ro \\
\hline laselvae & Occi-Cent & Trop-Suba & Basa-Suba & Trop-Suba & Inter & Co \\
\hline leucoblepharis & todas & Suba-Pára & Andi & Suba-Alto & Som-Exp & Co-Ma-Ro \\
\hline
\end{tabular}


APÉNDICE (Continuación)

\begin{tabular}{|c|c|c|c|c|c|c|}
\hline Especie & Cordilleras & Rang 1995 & Rodr 2006 & Combinación & Iluminación & Sustrato \\
\hline lineariloba & Orie-Sept & Suba-Pára & Andi & Suba & Som-Inter & Co-Ho \\
\hline lobarioides (ciano) & Cent-Orie-Sept & Andi & Andi & Andi & Inter & Co \\
\hline lobarioides (cloro) & Occi-Cent-Orie & Suba-Pára & Suba-Pára & Suba-Subp & Som-Inter & Co \\
\hline lumbschiana & Cent-Orie & Suba-Pára & Suba-Pára & Suba-Supe & Inter-Exp & $\mathrm{Co}-\mathrm{Ma}-\mathrm{Su}$ \\
\hline luteocyphellata & Occi-Cent-Orie & Suba-Andi & Suba-Andi & Suba-Andi & Som-Inter & Co \\
\hline luteola & Cent & Andi & Andi & Andi & Inter & Co \\
\hline macrocyphellata & todas & Suba-Pára & Suba-Pára & Suba-Pára & Inter & Co-Ro \\
\hline macrofuliginosa & Occi-Cent & Andi-Pára & Andi-Pára & Alto-Supe & Inter-Exp & Co \\
\hline macrogyalocarpa & Cent-Orie & Andi-Pára & Andi-Pára & Alto-Supe & Inter & $\mathrm{Co}-\mathrm{Ma}$ \\
\hline macrothallina (ciano) & Occi & Andi & Andi & Andi & Inter & Co \\
\hline macrothallina (cloro) & Cent-Orie-Sept & Suba-Pára & Suba-Andi & Suba-Pára & Inter & Co \\
\hline maculofuliginosa & Cent-Orie & Suba-Pára & Suba-Pára & Suba-Pára & Som-Exp & Co-Ro \\
\hline marilandia & Occi-Cent-Orie & Suba-Pára & Suba-Pára & Suba-Supe & Inter-Exp & $\mathrm{Co}-\mathrm{Su}$ \\
\hline microcyphellata & Occi-Cent-Orie & Suba-Pára & Suba-Pára & Suba-Pára & Som-Inter & Co-Ho \\
\hline microisidiata & Occi-Orie & Suba & Suba & Suba & Inter & Co \\
\hline minutula & Occi-Orie & Suba-Pára & Suba-Pára & Andi-Pára & Inter-Exp & Co \\
\hline neoboschiana & Cent-Orie & Pára & Pára & Pára & Inter & $\mathrm{Co}-\mathrm{Su}$ \\
\hline neolinearis & Occi-Orie & Andi & Andi-Pára & Andi-Pára & Som-Inter & $\mathrm{Co}-\mathrm{Su}$ \\
\hline neolinita & Occi-Cent-Orie & Suba-Pára & Suba-Pára & Suba-Pára & Inter & $\mathrm{Co}-\mathrm{Ma}$ \\
\hline neopulmonaria & Occi-Cent-Orie & Suba-Pára & Suba-Pára & Andi-Pára & Som-Inter & todos \\
\hline neopulmonarioides (ciano) & todas & Suba-Pára & Suba-Andi & Suba-Subp & Inter & Co-Ro \\
\hline neopulmonarioides (cloro) & Cent-Orie-Sept & Suba-Andi & Suba-Andi & Andi-Pára & Inter & Co-Ma \\
\hline ocaniensis & Occi-Cent-Orie & Suba-Pára & Suba-Pára & Suba-Subp & Inter & $\mathrm{Co}-\mathrm{Ma}$ \\
\hline orizabana & Cent-Orie-Sept & Suba-Pára & Andi-Pára & Suba-Pára & Inter-Exp & Co-Ho-Ro-Su-Br \\
\hline papillata & Occi-Orie & Suba-Andi & Suba-Andi & Andi-Alto & Inter & Co \\
\hline parahumboldtii & Occi-Cent-Orie & Andi-Pára & Andi-Pára & Alto-Pára & Som-Inter & $\mathrm{Co}-\mathrm{Su}$ \\
\hline paralimbata & Occi-Cent & Andi-Pára & Andi-Pára & Andi-Supe & Som-Inter & $\mathrm{Co}-\mathrm{Su}$ \\
\hline paramuna & Occi-Cent-Orie & Suba-Pára & Andi & Suba-Pára & Som-Inter & Co \\
\hline peltigerella & Orie & Andi & Andi & Andi-Pára & Som-Exp & Co-Ro-Br \\
\hline phyllidiata & Cent-Orie & Suba-Pára & Suba-Pára & Suba-Supe & Som-Inter & Co-Ma-Ro \\
\hline phyllidiodichotoma & Occi & Suba & Suba & Suba & Inter & $\mathrm{Co}$ \\
\hline phyllidiofuliginosa & Cent-Orie & Andi-Pára & Andi-Pára & Andi-Pára & Inter-Exp & $\mathrm{Co}-\mathrm{Su}$ \\
\hline phyllidiokunthii (ciano) & Cent-Orie & Suba-Pára & Suba-Pára & Suba-Pára & Inter-Exp & Co \\
\hline phyllidiokunthii (cloro) & Cent-Orie & Suba-Andi & Suba-Andi & Suba-Andi & Inter & $\mathrm{Co}-\mathrm{Ma}$ \\
\hline plumbeociliata & Cent-Orie & Suba-Andi & Suba-Andi & Suba-Subp & Inter-Exp & Co-Ro-Su \\
\hline pseudobeauvoisii & Occi-Cent-Orie & Suba-Pára & Suba-Pára & Suba-Subp & Inter-Exp & Co-Ma-Ro \\
\hline pseudolimbata & Occi-Orie & Suba-Pára & Suba-Pára & Suba-Pára & Som-Inter & $\mathrm{Co}-\mathrm{Su}$ \\
\hline pseudolobaria (ciano) & Occi & Andi & Andi & Andi & Inter & $\mathrm{Co}$ \\
\hline pseudolobaria (cloro) & todas & Suba-Pára & Suba-Pára & Suba-Pára & Som-Inter & Co-Ma-Ro \\
\hline pseudosylvatica & Occi-Cent & Suba-Andi & Suba-Andi & Suba-Andi & Inter & $\mathrm{Co}-\mathrm{Su}$ \\
\hline pulmonarioides & Occi-Cent & Suba-Pára & Suba-Pára & Suba-Pára & Inter & $\mathrm{Co}$ \\
\hline rhizinata & Occi-Cent & Suba-Pára & Suba-Pára & Suba-Pára & Som-Exp & $\mathrm{Co}-\mathrm{Su}-\mathrm{Br}$ \\
\hline robertiana & Occi-Cent-Orie & Suba-Pára & Suba-Pára & Suba-Subp & Inter & Co \\
\hline roseocyphellata & Occi-Cent-Orie & Suba-Andi & Suba-Andi & Suba-Subp & Som-Inter & Co-Ma-Ro-Su \\
\hline rubropruinosa & Occi & Suba & Suba & Suba & Inter & Co \\
\hline rudiuscula & Orie & Andi & Andi & Andi & Inter & Co \\
\hline scabrosa & Occi-Cent-Orie & Trop-Andi & Basa-Andi & Trop-Andi & Som-Exp & $\mathrm{Co}-\mathrm{Ma}-\mathrm{Su}-\mathrm{Br}$ \\
\hline
\end{tabular}


APÉNDICE (Continuación)

\begin{tabular}{lllllll}
\multicolumn{1}{c}{ Especie } & \multicolumn{1}{c}{ Cordilleras } & Rang 1995 & \multicolumn{1}{c}{ Rodr 2006 } & Combinación & Iluminación & Sustrato \\
silverstonii & Occi & Suba & Andi & Suba-Andi & Inter & Co \\
squamifera & Occi-Cent & Andi-Pára & Andi-Pára & Andi-Pára & Som-Inter & Co-Ro-Su \\
subdenudata & Occi-Cent-Sept & Suba-Pára & Andi-Pára & Suba-Pára & Som-Inter & Co \\
subfilicinella & todas & Suba-Pára & Suba-Andi & Suba-Subp & Inter & Co \\
sublimbatoides & Occi-Cent-Orie & Suba-Pára & Andi-Pára & Suba-Supe & Inter-Exp & Co-Ro-Su \\
subrudiuscula & Cent & Andi-Pára & Andi-Pára & Andi-Pára & Som-Inter & Co-Su \\
subscrobiculata & Cent-Orie & Suba-Andi & Suba-Andi & Suba-Subp & Inter & Co-Ma-Ho-Su \\
subscrobiculata (aff.) & Occi-Cent & Andi & Andi & Andi & Inter & Co \\
subtomentella (aff.) & Occi & Suba-Andi & Suba-Andi & Suba-Alto & Inter & Co \\
sylvatica & Cent-Orie & Andi-Pára & Suba-Pára & Suba-Pára & Inter-Exp & Co-Ro-Su \\
tatamana (ciano) & Occi & Andi & Andi & Andi & Inter & Co \\
tatamana (cloro) & Occi & Andi & Andi & Andi & Inter & Co \\
tolimensis & Cent & Andi-Pára & Andi-Pára & Andi-Pára & Som-Inter & Co-Ho \\
tomentosa & Occi-Cent-Orie & Suba-Andi & Suba-Andi & Suba-Alto & Som-Exp & Co-Ma-Ro \\
tunjensis & Orie & Suba-Andi & Suba-Andi & Suba-Alto & Inter & Ma-Su \\
venosa (aff.) & Occi & Suba & Suba & Suba & Inter & Co \\
viviana & Orie & Andi & Andi & Andi-Subp & Exp & Co-Ro \\
weigelii & Cent-Orie & Suba-Pára & Suba-Pára & Suba-Supe & Som-Inter & Co-Ma-Ro-Su \\
weigelii (aff.) & Occi-Cent-Orie & Trop-Pára & Basa-Pára & Trop-Pára & Som-Inter & Co-Ma-Ho-Ro-Su \\
\hline
\end{tabular}

Cent $=$ Cordillera Central, Occi $=$ Cordillera Occidental, Orie $=$ Cordillera Oriental; Alto $=$ Altoandino, Andi $=$ Andino, Pára $=$ Páramo, Suba $=$ Subandino, Subp $=$ Subpáramo, Supe $=$ Superpáramo, Trop $=$ Tropical; Som $=$ sombreada, Inter $=$ Intermedia, Exp = Expuesta; $\mathrm{Br}=$ Briófitos, $\mathrm{Ho}=$ Hojarasca, $\mathrm{Co}=$ Corteza, $\mathrm{Ma}=$ Madera, Ro $=$ Roca, $\mathrm{Su}=$ Suelo. 